\title{
Structural Damage Assessment by Using Stiffness-based Method and Cubic Spline Interpolation
}

\author{
Wei-Chih $\mathrm{Su}^{1, *}$, Chiung-Shiann Huang ${ }^{2}$, and Jyh-Horng $\mathrm{Wu}^{1}$ \\ ${ }^{1}$ National Center for High-performance Computing, National Applied Research Laboratories, No. 7 R\&D $6{ }^{\text {th }}$ Rd Hsinchu, Taiwan \\ ${ }^{2}$ Department of Civil Engineering, National Chiao Tung University, 1001 University Rd Hsinchu, Taiwan
}

\begin{abstract}
This study explores the possibility of using stiffness-based method and cubic spline interpolation to locate the damaged storey of a building during a strong earthquake, and corresponding stiffness matrix of structure often change in the earthquake process. The time series model of a building is established from the full structural dynamic responses. Next, the coefficient matrix of the time series model could be solved by recursive least squares (RLS) algorithms. Then, the model parameters of a building are calculated by the coefficient matrix of time series model. Finally, the identified natural frequencies and mode shapes of structure that corrected by cubic spline interpolation would be used to construct the stiffness matrix of a building. Then, the damage location of a building could be detected by the identified stiffness matrix of a building. The effectiveness of the proposed procedure is verified using numerically simulated earthquake responses of the finite element model of a six-storey frame.
\end{abstract}

\section{Introduction}

It is essential that detect the damaged location by using the identified modal parameters of an existing structure or bridge from the measured dynamical responses in the field of structural health monitoring. System identification is a process to create mathematical models of structures using measurements of the dynamic responses and base excitation of structure. Based on the properties of coefficient estimation approaches, the process of system identification are divided into two broad categories, online and offline estimation.

- Online estimation algorithms estimate the coefficient matrix of time series model when the new responses are measureable during the dynamic process. These algorithms including: recursive least square approaches [1-3]; recursive gradient approaches $[4,5]$ and recursive maximum likelihood approaches $[6$, 7].

- Offline estimation algorithms estimate the coefficient matrix of time series model after collecting all the input and output data. These algorithms including: least square approaches [8]; maximum likelihood approaches [9]; artificial neural network [10]; genetic algorithm [11].

The vibration-based damage detection method would use to locate the damaged sotreys based on examining change in measured dynamic responses. Stiffness matrix based process requires as follows: 1. identifying the modal parameters of structures; 2. constructing the stiffness matrix of structures by identified modal parameters; 3 . computing the variation of the identified stiffness matrix between the damaged and undamaged structure.

This study utilizes a simple and efficient modal identification method and stiffness-based damage locating approach to determine which floors of a structure exhibit a change in stiffness. First, the time series model of a structure is established and the recursive least square approach is used to estimate the coefficient matrix of time series model. Next, the modal parameters of structure would be calculated by the estimated coefficient matrix of time series model. Then, the stiffness matrix of structure could be obtained from the identified modal parameters. However, it is difficult to observe the dynamic responses of all degree of freedoms. In here, the cubic spline interpolation would be used to obtain the mode shapes of the unobserved degree of freedom from the identified modal parameters.

The proposed procedure is validated using the simulated earthquake acceleration responses of a sixstory finite element model. The incomplete measurement situation would be assumed and the cubic spline interpolation would be used to calculate the mode shapes of the unobserved degree of freedom.

\section{Methodology}

A building structure could be considered with viscous damping. The dynamic responses of building must

Corresponding author: wichcv86@gmail.com 
satisfy the equation of motion, and could be described as follows

$$
M+C \&+K x=\mathbf{f}
$$

where $\mathbf{M}, \mathbf{C}$ and $\mathbf{K}$ are mass, damping and stiffness matrices, respectively; $\&$ and $\mathbf{x}$, respectively, are the acceleration, velocity and displacement responses vectors of the building, and $\mathbf{f}$ is the input force vectors. According to the central difference approach, equation of motion could be rewrite by time series model

$$
\mathbf{y}_{t}=\sum_{i=1}^{I} \boldsymbol{\Phi}_{i} \mathbf{y}_{t-i \Delta t}+\sum_{j=0}^{J} \boldsymbol{\Theta}_{j} \mathbf{f}_{t-j \Delta t}
$$

where, $\mathbf{y}_{t-i \Delta t}$ and $\mathbf{f}_{t-i \Delta t}$ are the vectors of measured responses and input forces at time $t-i \Delta t$, respectively; $1 / \Delta t$ is the sampling rate of the measurement, $\Phi_{i}$ and $\Theta_{j}$ are matrices of coefficient functions to be determined in the model. Rearranging Eq.(2) yields

$$
\mathbf{y}_{t}=\bar{\varphi}_{t}^{\mathbf{T}} \theta_{t}
$$

where

$$
\left\{\begin{array}{l}
\bar{\varphi}_{t}=\left[\begin{array}{llllll}
\mathbf{y}_{t-\Delta t} & \cdots & \mathbf{y}_{t-1 \Delta t} & \mathbf{f}_{t} & \cdots & \mathbf{f}_{t-j \Delta t}
\end{array}\right]^{\mathbf{T}} \\
\boldsymbol{\theta}_{t}=\left[\begin{array}{llllll}
\Phi_{t, 1} & \cdots & \Phi_{t, t} & \Theta_{t, 0} & \cdots & \Theta_{t, j}
\end{array}\right]
\end{array}\right.
$$

A recursive least square algorithm can be used to estimate and track the time-variant coefficient matrix of time series model as follows

$$
\left\{\begin{array}{l}
\bar{\theta}_{t+1}=\bar{\theta}_{t}+L_{t} \varepsilon_{t} \\
\varepsilon_{t}=y_{t}-\bar{\varphi}_{t}^{T} \bar{\theta}_{t} \\
L_{t}=P_{t} \bar{\varphi}_{t}=\frac{P_{t-1} \bar{\varphi}_{t}}{\lambda+\bar{\varphi}_{t}^{T} P_{t-1} \bar{\varphi}_{t}} \\
P_{t}=\frac{1}{\lambda}\left[P_{t-1}-L_{t} \bar{\varphi}_{t}^{T} P_{t-1}\right]
\end{array}\right.
$$

where, $\boldsymbol{L}_{\boldsymbol{t}}$ is the filtering gain; $\boldsymbol{\varepsilon}_{\boldsymbol{t}}$ is the a priori prediction error; $\boldsymbol{P}_{\boldsymbol{t}}$ is the estimation covariance matrix and $\lambda$ is the forgetting factor.

Eq. (2) reveals that the modal parameters of a structure are determined from $\Phi_{i}$ with $i=1,2, \ldots, I$. A matrix $\mathrm{G}$ is constructed from $\Phi_{i}$ as

$$
\mathbf{G}=\left[\begin{array}{cccccc}
\mathbf{0} & \mathbf{I} & \mathbf{0} & \mathbf{0} & \mathrm{L} & \mathbf{0} \\
\mathbf{0} & \mathbf{0} & \mathbf{I} & \mathbf{0} & \mathrm{L} & \mathbf{0} \\
\mathrm{M} & \mathrm{M} & \mathrm{M} & \mathrm{M} & \mathrm{L} & \mathrm{M} \\
\boldsymbol{\Phi}_{I} & \boldsymbol{\Phi}_{I-\mathbf{1}} & \boldsymbol{\Phi}_{I-2} & \boldsymbol{\Phi}_{I-3} & \mathrm{~L} & \boldsymbol{\Phi}_{\mathbf{1}}
\end{array}\right]_{\bar{N} \times \bar{N}}
$$

Consider the response of a structure described by equation of motions such as Eq. (1). Assuming properties damping, the orthogonality property of the mode shapes with respect to the mass and stiffness matrix leads to [13]

$$
\mathbf{M}_{D}=\varphi^{\mathrm{T}} \mathbf{M} \boldsymbol{\varphi} ; \quad \mathbf{K}_{D}=\varphi^{\mathrm{T}} \mathbf{K} \boldsymbol{\varphi}
$$

in which $\mathbf{M}_{D}$ and $\mathbf{K}_{D}$ are the diagonal modal mass matrix and diagonal modal stiffness matrix, respectively. The equation used to express the square of modal frequencies as a matrix is

$$
\boldsymbol{\Lambda}=\mathbf{M}_{D}^{-\mathbf{T} / 2} \mathbf{K}_{D} \mathbf{M}_{D}^{-1 / 2}=\mathbf{M}_{D}^{-\mathbf{T} / 2} \boldsymbol{\varphi}^{\mathrm{T}} \mathbf{K} \boldsymbol{\varphi} \mathbf{M}_{D}^{-1 / 2}
$$

Assuming the mass matrix was given, then, the stiffness matrix could be obtained

$$
\mathbf{K}=\boldsymbol{\varphi}^{-\mathbf{T}} \mathbf{M}_{D}^{\mathbf{T} / 2} \boldsymbol{\Lambda} \mathbf{M}_{D}^{1 / 2} \boldsymbol{\varphi}^{-1}
$$

\section{Numerical Verification}
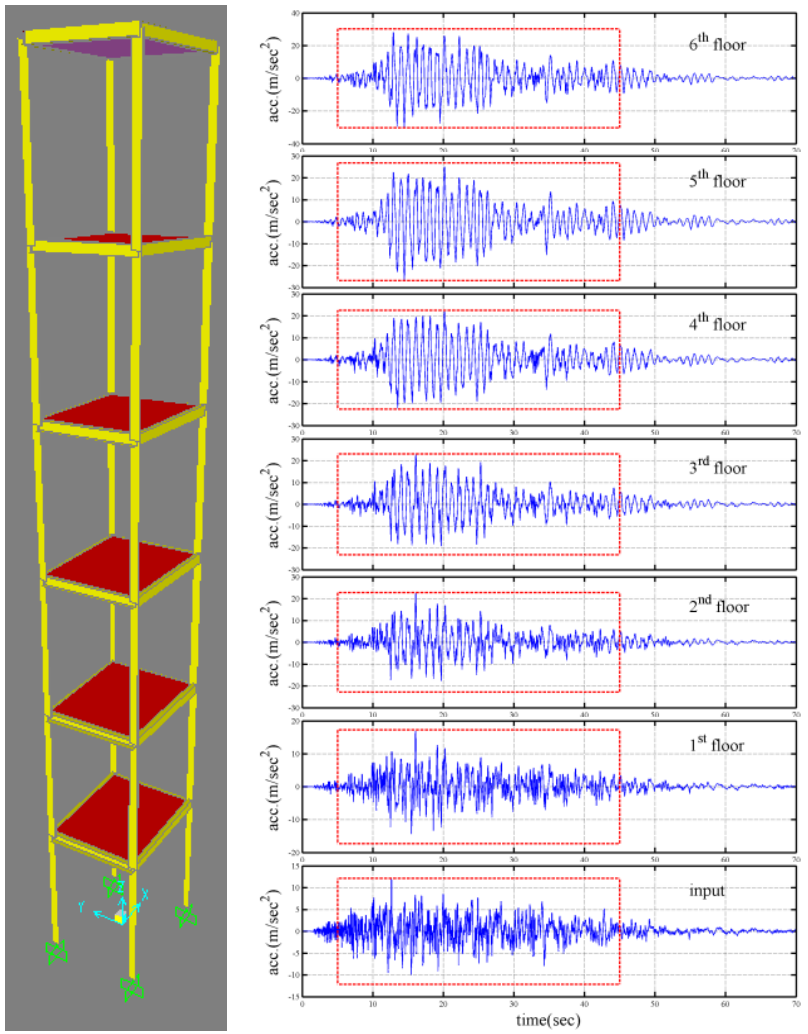

Fig. 1. Schematic diagram of a six-storey finite element model and its simulation responses

The finite element model of six-storey frame was built to demonstrate the feasibility of the proposed procedure, and this frame was subjected to a simulated 1999 Chi-chi earthquake at its base in $\mathrm{X}$ direction (see Fig. 1). The geometric parameter of this six-storey frame were $25 \mathrm{~cm}$ long, $20 \mathrm{~cm}$ wide and $180 \mathrm{~cm}$ high. The $5 \%$ of the modal damping ratio would be assumed during the simulation process. Plates were fixed on each floor, such that the total mass of frame at each floor was approximately $6.732 \mathrm{~kg}$. The columns have rectangular section of $2 \mathrm{X} 18$, with units is $\mathrm{mm}$. Table 1 shows the theoretical modal parameters. This frame was subjected to base excitations of the 1999 Chi-chi earthquake. The acceleration responses of the base and all floors at $\mathrm{t}=5-45$ seconds were used in evaluating modal parameters for the frame. Fig. 1 displays the time history of base excitation and acceleration responses in $\mathrm{X}$ direction of this numerical model subject the Chi-chi earthquake. 
Table 1. Theoretical modal parameters of numerical model

\begin{tabular}{|c|cccccc|}
\hline modal & $1^{\text {st }}$ & $2^{\text {nd }}$ & $3^{\text {rd }}$ & $4^{\text {th }}$ & $5^{\text {th }}$ & $6^{\text {th }}$ \\
\hline \hline$f_{n}(\mathrm{~Hz})$ & 0.966 & 2.842 & 4.555 & 6.000 & 7.095 & 7.778 \\
\hline \hline$\xi(\%)$ & 5.000 & 5.000 & 5.000 & 5.000 & 5.000 & 5.000 \\
\hline \hline & 1.000 & -0.947 & -0.843 & -0.685 & -0.484 & -0.252 \\
& 0.942 & -0.478 & 0.225 & 0.818 & 1.000 & 0.675 \\
$\boldsymbol{\varphi}$ & 0.830 & 0.233 & 1.000 & 0.481 & -0.658 & -0.945 \\
& 0.668 & 0.825 & 0.477 & -0.939 & -0.249 & 1.000 \\
& 0.468 & 1.000 & -0.665 & -0.247 & 0.942 & -0.828 \\
& 0.241 & 0.668 & -0.944 & 1.000 & -0.827 & 0.468 \\
\hline
\end{tabular}

\subsection{Incomplete measurement}

It is difficult to observe the total degree of freedom in the site experiment. To somewhat simulated this situation, the dynamic responses of the second story wouldn't be used to the identification process of modal parameters. To check for agreement between the identified mode shapes and theoretical ones, the modal assurance criterion (MAC) proposed by Allemang and Brown [14] was used, which was defined as

$$
\operatorname{MAC}\left(\boldsymbol{\varphi}_{k, i}, \boldsymbol{\varphi}_{k, a}\right)=\frac{\left|\boldsymbol{\varphi}_{k, i}^{\mathrm{T}} \boldsymbol{\varphi}_{k, a}\right|^{2}}{\boldsymbol{\varphi}_{k, i}^{\mathrm{T}} \boldsymbol{\varphi}_{k, i} \boldsymbol{\varphi}_{k, a}^{\mathrm{T}} \boldsymbol{\varphi}_{k, a}}
$$

where $\varphi_{k, i}$ is the identified $i^{\text {th }}$ mode shape and $\varphi_{k, a}$ is the corresponding analytical mode shape. The value of MAC is between 0 and 1 . When the two mode shapes are more similar, the resultant value of MAC is closer to one. In other words, when two mode shapes are orthogonal with each other, the resultant MAC value is zero.

The acceleration responses of the base and all degree of freedom at $\mathrm{t}=5-45 \mathrm{sec}$ would be used to identify modal parameters. Figure 2(a) plots the results obtained from processing the simulated input and acceleration responses of all stories by using recursive least square approach. In this study, the forgetting factor would be given as 0.995 . In the modal identification process, the accurately and stable modal parameters were obtained.

\subsection{Constructing stiffness matrix}

In the stiffness matrix construction process, the identified mode shapes of all degree of freedom is necessary. In here, the cubic spline interpolation would be used to calculate the mode shapes of the unobserved degree of freedom. Under the assumption of given mass matrix, combined with the identified natural frequencies and mode shapes, the stiffness matrix of structure can be determined by Eq. (9). Table 2 shows the theoretical stiffness matrix of the six-storey frame extracted from finite element model. Due to the values of off-tridiagonal elements less than $1 \%$ of the tridiagonal elements, in the follow numerical study, the relative errors of the tridiagonal element would be discussed.

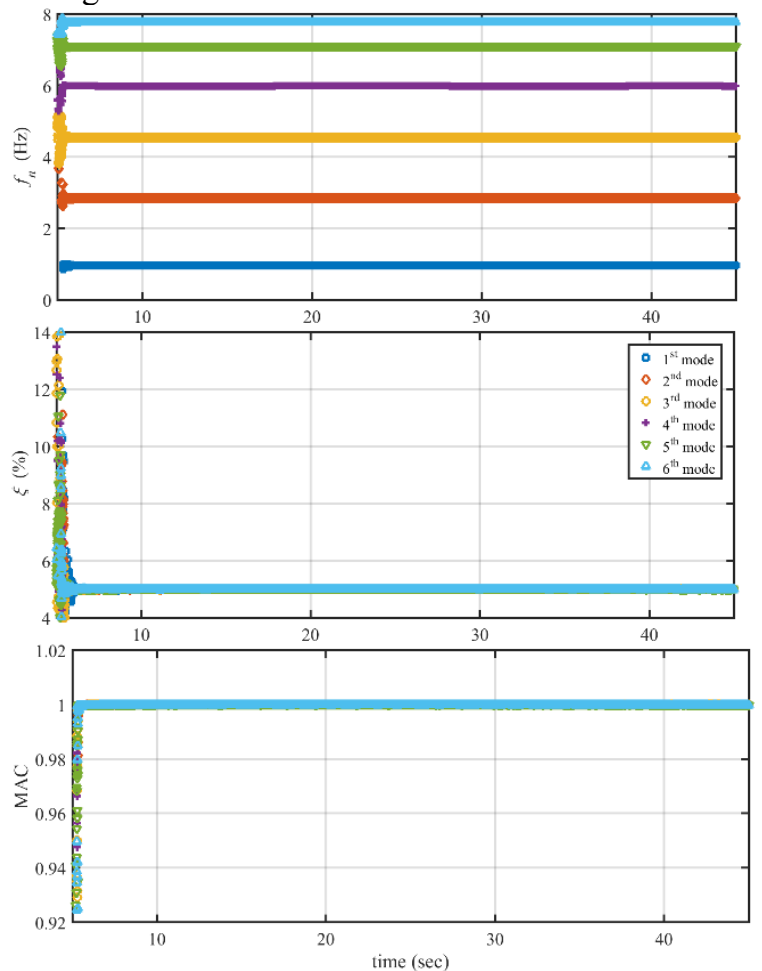

(a)

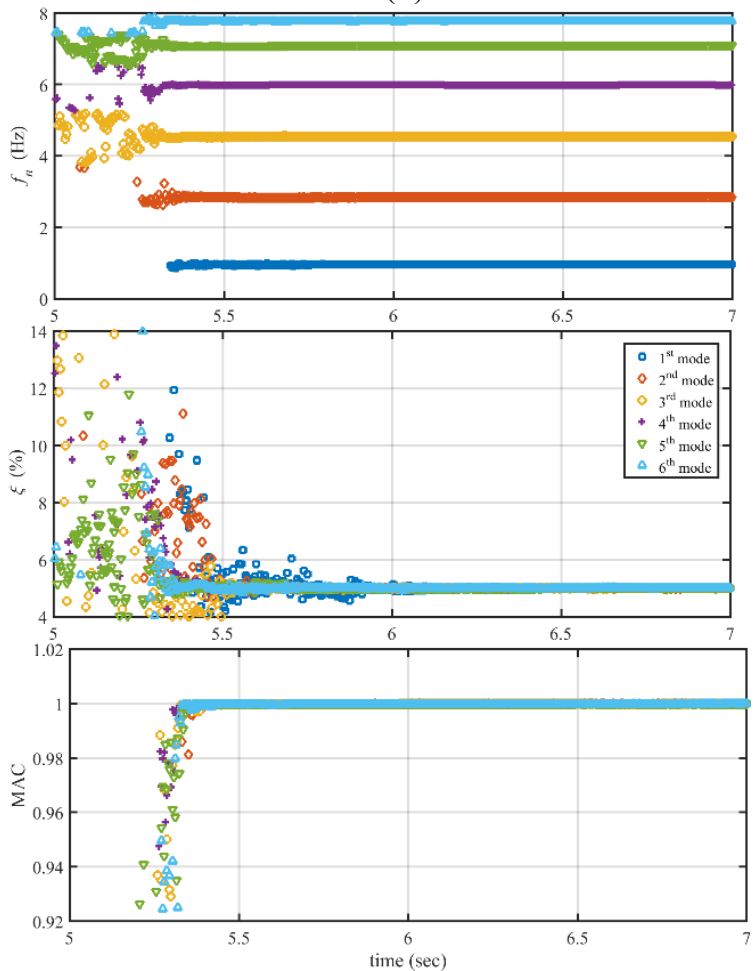

(b)

Fig. 2. The identified instantaneous modal parameters (a) $t=5$ $45 \mathrm{sec}$; (b) $\mathrm{t}=5-7 \mathrm{sec}$

Table 3 shows the relative errors of the calculated stiffness matrix using Eq. (9). The modal parameters that were identified from the simulation dynamic responses of certain observed degree of freedom would be used in Eq. (9). For the identified results from the full measurement, the maximum relative error is $4.221 \%$. It 
is evidently that the variant of the local stiffness less than $5 \%$ is difficult to detect from the identified modal parameters.

Table 2. The theoretical stiffness matrix of the six-storey frame

\begin{tabular}{|cccccc|}
\hline \multicolumn{6}{|c|}{ K matrix (N/cm) } \\
\hline \hline 42.480 & -42.585 & 0.067 & 0.011 & 0.011 & 0.011 \\
-42.585 & 85.180 & -42.652 & 0.057 & 0.000 & 0.000 \\
0.067 & -42.652 & 85.181 & -42.652 & 0.057 & 0.000 \\
0.011 & 0.057 & -42.652 & 85.181 & -42.652 & 0.057 \\
0.011 & 0.000 & 0.057 & -42.652 & 85.181 & -42.652 \\
0.011 & 0.000 & 0.000 & 0.057 & -42.652 & 85.237 \\
\hline
\end{tabular}

Next, for the identified results from the incomplete measurement, the cubic spline could be used to calculate the deformations of the unobserved degree of freedom in each modal. The maximum relative error is $4.725 \%$. It is evidently that the variant of the local stiffness more than $5 \%$ is easy to detect from the identified modal parameters.

Table 3. The relative stiffness matrix errors on tridiagonal (unit:\%)

\begin{tabular}{|c|cccccc|}
\hline \multicolumn{7}{|c|}{ without mode shapes correction } \\
\hline \hline \multirow{5}{*}{ Full } & 4.221 & 3.718 & $\backslash$ & $\backslash$ & $\backslash$ & $\backslash$ \\
measurement & 3.718 & 2.650 & 0.950 & $\backslash$ & $\backslash$ & $\backslash$ \\
& $\backslash$ & 0.950 & 3.145 & 2.074 & $\backslash$ & $\backslash$ \\
& $\backslash$ & $\backslash$ & 2.074 & 2.360 & 2.065 & $\backslash$ \\
& $\backslash$ & $\backslash$ & $\backslash$ & 2.065 & 1.509 & 0.292 \\
& $\backslash$ & $\backslash$ & $\backslash$ & $\backslash$ & 0.292 & 0.765 \\
\hline \hline \multirow{5}{*}{ Incomplete } & 4.725 & 3.494 & $\backslash$ & $\backslash$ & $\backslash$ & $\backslash$ \\
& 3.494 & 2.169 & 3.447 & $\backslash$ & $\backslash$ & $\backslash$ \\
& $\backslash$ & 3.447 & 2.944 & 1.716 & $\backslash$ & $\backslash$ \\
& $\backslash$ & $\backslash$ & 1.716 & 3.289 & 2.266 & $\backslash$ \\
& $\backslash$ & $\backslash$ & $\backslash$ & 2.266 & 3.851 & 0.629 \\
& $\backslash$ & $\backslash$ & $\backslash$ & $\backslash$ & 0.629 & 1.962 \\
\hline \hline
\end{tabular}

\section{Conclusions}

This study extended cubic spline interpolation for creating the stiffness matrix of building from structural dynamic responses. The procedure of stiffness matrix creation from the measured vibration would be divided three stages, 1 . The modal parameters of $\mathrm{s}$ structure would be identified from measured seismic responses; 2 . Assuming the mass properties was given and calculating the deformations of the unobserved degree of freedom in each modal via cubic spline interpolation; 3 . Constructing the stiffness from the identified natural frequencies and corrected mode shapes.
In modal identification stage, the time series model was constructed in time domain. The time-varying coefficients in time series model were determined by a recursive least square approach. Then, the modal parameters of the structure were calculated from these coefficients. For incomplete measurement case, it is difficult to construct the stiffness matrix from the identified modal parameters. Therefore, the cubic spline interpolation would be used to calculate the deformation of the unobserved degreed of freedom in each modal. Finally, the stiffness matrix of the structure would be constructed via the identified natural frequencies and corrected mode shapes.

The numerically simulated responses of a six-storey finite element model subjected earthquake input were used to validate the proposed procedure. The effects of forget factor on the ability to identify the modal parameters were also investigated. The recursive least square approach apply on full measurement data did provide more accurately stiffness than the process incomplete measurement data.

The authors would like to thank the Ministry of Science and Technology of the Republic of China, Taiwan, for financially supporting this research under Contract No. MOST 106-2622M-492-001-CC2.

\section{References}

1. S. Ljung, L. Ljung, Automatica, 21 (1985)

2. A. Vahidi, A. Stefanopoulou, H. Peng, Veh Mec Mobil, Int J. 43, 31-55 (2005)

3. F. Ding; Y. Wang, J. Ding, Dig Signal Proc, 37, 100-108 (2015)

4. S.I. Adachi; A. Sano, Elec Com. Japan, 69, 1-9 (1986)

5. H. Oku, H. Kimura, Automatica, 38, 1035-1043 (2002).

6. M. Drton, M. Eichler, T.S. Richardson, J. Mach Learn Res, 10, 2329-2348 (2009)

7. T. Fujiwara, H. Yamamoto, T. Kasami, Shu Lin, IEEE Trans Inf Theo, 44, 714-729 (1998)

8. W.C. Su, C.S. Huang, C.H. Chen, C.Y. Liu, H.C. Huang, Q.T. Le, Comp Aid Civ Infras Eng, 29, 738757 (2014)

9. J.H Li, F. Ding, G.W Yang, Math Comp Model, 55, 442-450 (2012)

10. C.S. Huang, S.L. Hung, C.M. Wen, T.T. Tu, Earth Eng Struct Dyn, 32, 187-206 (2002)

11. T. Kumon, T. Suzuki, M. Iwasaki, M. Matsuzaki, N. Matsui, S. Okuma, Elec Eng. Japan, 142, 45-55 (2003)

12. C.S. Huang, J. Sound Vib, 241, 337-359 (2001)

13. J.W. Tedesco, W.G. McDougal, C.A. Ross (Menlo Park, Calif, 1999).

14. R.J. Allemang, D.L. Brown. Mod Analy, Proc Int Conf. 110-116 (1982) 